\title{
Gender-related differences in cardiovascular risk profile in black patients with diabetes mellitus
}

\begin{abstract}
Background: Women with diabetes mellitus are at higher risk of cardiovascular disease compared to men and this may be a result of greater clustering of risk factors among women. This study aimed to assess gender differences in cardiovascular risk factors among adults with diabetes mellitus.

Methods: This hospital-based cross-sectional study involved 100 patients with diabetes. Fifty individuals without diabetes matched for gender were selected for comparison. Blood samples were analyzed for fasting glucose, lipid parameters and serum creatinine.

Results: The patients with DM were significantly older than those without, with higher body mass index, blood pressures, lower HDL cholesterol and lower eGFR. Among those with diabetes, females had higher blood pressures and body mass index (BMI) but significantly lower eGFR compared to males. There was a significant negative correlation between duration of diabetes and eGFR in females $(r=-0.544, p<0.001)$ but not in males $(r=-0.271, p=0.065)$. On multiple regressions, age and gender were significant independent predictors of declining eGFR. Overall less than one third of the patients with DM were at the recommended target for BMI, 35\%achieved fasting blood glucose target with significantly fewer women compared to men achieving this $(\mathrm{p}=0.005)$. Thirty four percent achieved LDL target with no significant difference between males and females. Significantly fewer women achieved the BP and HDL targets compared to men.
\end{abstract}

Conclusion: This indicates overall poor risk factor control but worse among women, emphasizing the need for better implementation of guideline recommendations for management of diabetes to reduce future cardiovascular disease.
Volume 2 Issue 2 - 2015

\author{
Sandra Ofori, Chinyere Wachukwu \\ Department of Internal Medicine, University of Port Harcourt, \\ Rivers state, Nigeria
}

\author{
Correspondence: Sandra Ofori, Department of Medicine, \\ University of Port Harcourt, East-West road Choba, Rivers \\ state, Nigeria, Tel +2348096598508, \\ Email sandytom77@yahoo.com
}

Received: January 14, 2015 | Published: April 17, 2015

Keywords: diabetes mellitus, risk factors, gender differences

Abbreviations: DM, diabetes mellitus; IDF, international diabetes federation; NCDs, non-communicable diseases; CVD, cardiovascular disease; GFR, glomerular filtration rate; SPSS, statistical package for social sciences; LDL, low-density lipoprotein; HDL, high density lipoprotein; SBP, systolic blood pressure; DBP, diastolic blood pressure; LDLC, low density lipoprotein cholesterol; HDLC, high density lipoprotein cholesterol; FPG, fasting plasma glucose; eGFR, estimated glomerular filtration rate; TC, total cholesterol; BMI, body mass index; BP, blood pressure; TG, triglycerides

\section{Introduction}

Diabetes mellitus (DM) is a growing problem around the world. The international diabetes federation (IDF) estimates that 387 million people have DM globally, of which about $46.3 \%$ are undiagnosed. This figure is expected to reach 205 million people by 2035 if nothing is done to stem the tide. ${ }^{1}$ Health in sub-Saharan Africa has been traditionally dominated by infectious disease. However, with rapid urbanization, non-communicable diseases (NCDs) like DM are quickly becoming a new priority for health in the region. The Africa Region also has the highest proportion of undiagnosed diabetes, at least $78 \%$. An estimated 344,000 deaths in the region can be attributed to diabetes. This represents $6.1 \%$ of deaths from all causes. 1

In Nigeria, over the past 30years the prevalence of DM has been increasing steadily. In 1971, a hospital survey by Ugoya et al. $^{2}$ in
Ibadan estimated a prevalence of $0.43 \%$. The Federal Ministry of Health data collected in 1998, recorded a national prevalence rate of diabetes of 2.2 percent, which translated to over 2 million persons living with the disease at the time. ${ }^{3}$ Currently the most recent IDF estimate puts the prevalence of DM in Nigeria at $4.64 \% .^{1}$ In Port Harcourt, a cosmopolitan city in Nigeria, the prevalence was found to be $6.8 \%$ in the general population in a study by Nyenwe et al. ${ }^{4}$

The major complications of DM are microvascular disease and macrovascular disease. Diabetes is a major risk factor for cardiovascular disease (CVD) including coronary heart disease, stroke and peripheral artery disease. The risk of cardiovascular disease increases with age in both men and women. Premenopausal women have a lower risk of cardiovascular disease when compared to men however this is attenuated by the presence of T2DM. ${ }^{5}$ Hyperglycaemia, the key metabolic derangement in DM only partly explains this risk, as it is more a predictor of microvascular events with a modest association with macrovascular complications. ${ }^{6}$ Sedentary lifestyle, obesity, cigarette smoking, hypertension and dyslipidemia are independent CVD risk factors that are commonly associated with DM.?

Women with T2DM have a greater risk of CVD when compared to men and this may be due to greater clustering of CVD risk factors in women with DM. ${ }^{5}$ Therefore this study set out to assess the gender specific differences in cardiovascular risk factors among individuals with T2DM without prevalent CVD. 


\section{Methodology}

This was a cross sectional study conducted in the medical outpatient clinic of the University of Port Harcourt teaching hospital. The study population consisted of adult black Africans who live in Port Harcourt, a bustling metropolis in the oil-rich South-South Nigeria.

A) Inclusion criteria consisted of i) adults over 30years with a known diagnosis of type 2 diabetes mellitus or on glucose lowering medication or two fasting blood glucose samples $>7.0 \mathrm{mmol} / 1$; ii) those who gave informed consent.

B) Exclusion criteria were i) adults over 30years but with known diagnosis of type 1 diabetes mellitus or who require insulin for survival; ii) adults who had a past history of stroke, transient ischaemic attack, angina pectoris or myocardial infarction.

A total of 100 patients with T2DM met the inclusion criteria. Fifty healthy non-diabetic individuals matched for gender who also gave consent were selected from staff and relatives of other patients and were included in this study as controls for comparison.

A brief history was used to ascertain demographic data including age, gender, smoking and alcohol history, family and personal history of hypertension, diabetes and cardiovascular disease. Blood pressure was measured with a standard (Accoson) mercury sphygmomanometer on the patients' right arm in the seated position with feet on the floor after a five-minute rest. Systolic and diastolic blood pressures were taken at Korotkoff phases 1 and 5 respectively to the nearest $2 \mathrm{mmHg}$. The average of two blood pressure measurements taken 5minutes apart was used. ${ }^{8}$

Weight was measured in kilograms to the nearest $0.5 \mathrm{~kg}$ with a mechanical weighing scale with the subject wearing only light clothing. Height was measured using a stadiometer with the subject standing feet together without shoes or head gear, back and heel, together against a vertical ruled bar to which a movable attached horizontal bar was brought to the vertex of the head and reading taken to the nearest $0.5 \mathrm{~cm}$. Body mass index was calculated as body weight in kilograms divided by the square of the height in meters. ${ }^{9}$

Blood samples were collected and analyzed in the chemical pathology laboratory of this hospital for fasting blood glucose, lipid profile and serum creatinine. Serum creatinine was used to calculate the estimated glomerular filtration rate (GFR) using the CockcroftGault formula. ${ }^{9}$ Low-density lipoprotein (LDL) cholesterol values were calculated using the Friedewald equation when the triglyceride level was less than 4.0mmol/1: $L D L=T C-\left(\frac{H D L+T G}{2.2}\right) .{ }^{10}$

Risk factor targets were defined using the international diabetes federation (IDF) global guideline for type 2 diabetes as follows: ${ }^{11}$
i. Body mass index $<25 \mathrm{~kg} / \mathrm{m}^{2}$
ii. Blood pressure $\leq 130 / 80 \mathrm{mmHg}$
iii. Fasting plasma glucose $<6.5 \mathrm{mmol} / 1$
iv. LDL cholesterol $<2.0 \mathrm{mmol} / 1$
v. HDL cholesterol $<1.0 \mathrm{mmol} / 1$
vi. Triglycerides $<2.3 \mathrm{mmol} /$

\section{Statistical analysis}

The data presented here was analyzed using statistical package for social sciences (SPSS) version 21.0 analytic software. Continuous variables were compared with the independent samples T-test and the Chi-square test was used to compare categorical variables. Gender differences were noted among the patients with diabetes and Pearson correlation was used to analyse the relationship between eGFR and duration of diabetes among males and females adjusting for confounders like body mass index, systolic and diastolic blood pressure. Multiple linear regression analysis was used to determine which variables significantly predicted a decline in eGFR among the patients with diabetes. All tests were considered to be statistically significant at the two-sided $\mathrm{P}$-value $\leq 0.05$.

\section{Results}

The patients with DM were significantly older than the controls, with a higher body mass index, higher systolic and diastolic blood pressures. They also had higher pulse pressure, lower HDL cholesterol and lower eGFR (Table 1). When analyzed by gender, the only significant differences among the male and female subjects without diabetes were with regards to systolic and diastolic blood pressures and serum triglycerides where males had higher values than females. On the other hand, among those with diabetes, females had higher blood pressures compared to males, in addition to higher BMI and significantly lower eGFR (Table 2).

Table I Baseline characteristics of the patient population

\begin{tabular}{|c|c|c|c|}
\hline Variable & $\begin{array}{l}\text { Patients with } \\
\text { DM } n=100\end{array}$ & $\begin{array}{l}\text { Controls } \\
n=50\end{array}$ & $P$ value \\
\hline Age & $53.4(10.4)$ & $43.5(9.5)$ & $<0.001$ \\
\hline Female n (\%) & $53(53)$ & $26(52)$ & 0.908 \\
\hline Duration of DM (years) & $6.7(6.3)$ & - & - \\
\hline Body mass index $\left(\mathrm{kg} / \mathrm{m}^{2}\right)$ & $28.7(5.1)$ & $26.1(3.3)$ & 0.001 \\
\hline SBP mmHg & $129.3(18.0)$ & II $5.7(10.4)$ & $<0.001$ \\
\hline DBP $\mathrm{mmHg}$ & $78.1(10.7)$ & $74.4(7.3)$ & 0.03 \\
\hline Pulse pressure $\mathrm{mmHg}$ & $51.2(12.4)$ & $41.2(7.5)$ & $<0.001$ \\
\hline Total cholesterol (mmol/l) & $4.8(0.9)$ & $4.9(0.6)$ & 0.382 \\
\hline Triglycerides $(\mathrm{mmol} / \mathrm{l})$ & $1.3(0.5)$ & I.3 $(0.4)$ & 0.415 \\
\hline LDL (mmol/l) & $3.0(0.9)$ & $2.8(0.6)$ & 0.23 \\
\hline $\mathrm{HDL}(\mathrm{mmol} / \mathrm{l})$ & $1.2(0.4)$ & I.4 (0.4) & 0.001 \\
\hline FPG $(\mathrm{mmol} / \mathrm{l})$ & $8.8(4.0)$ & $4.8(0.5)$ & $<0.001$ \\
\hline eGFR (mls/min) & $91.6(0.7)$ & $109.7(11.9)$ & $<0.001$ \\
\hline
\end{tabular}

Data expressed as means (standard deviation)

DM, diabetes mellitus; SBP, systolic blood pressure; DBP, diastolic blood pressure; LDL, low density lipoprotein cholesterol; HDLC, high density lipoprotein cholesterol; FPG, fasting plasma glucose; eGFR, estimated glomerular filtration rate

Duration of diabetes was longer among females and although this was not statistically significant, there was a significant negative correlation between duration of diabetes and eGFR in females $(r=-$ $0.544, \mathrm{p}<0.001)$ but not in males $(\mathrm{r}=-0.271, \mathrm{p}=0.065)$ (Figures $1-2$ ). This remained significant after controlling for BMI, SBP and DBP (females $\mathrm{r}=-0.381, \mathrm{p}=0.007$; males $\mathrm{r}=-0.104, \mathrm{p}=0.509$ ). Multiple regression was performed to predict eGFR from gender, age, BMI, 
SBP and DBP. These variables combined, statistically significantly predicted eGFR, $\mathrm{F}(5,94)=18.5$, p $0<.000, \mathrm{R} 2=47.2$. However, only age and gender were significant independent predictors of declining eGFR (Table 3).

Table 2 Sex-related differences in the characteristics of the patients with and without DM

\begin{tabular}{|c|c|c|c|c|c|c|}
\hline \multirow{2}{*}{ Variable } & \multicolumn{3}{|c|}{ Patients with DM } & \multicolumn{3}{|l|}{ Controls } \\
\hline & Males $n=47$ & Females $n=53$ & $P$ value & Males n=24 & Females $n=26$ & $P$ value \\
\hline Age (years) & $54.1(10.2)$ & $52.1(10.6)$ & 0.356 & $42.3(8.4)$ & $45.6(10.4)$ & 0.194 \\
\hline DM duration (years) & $6.2(5.6)$ & $7.1(6.9)$ & 0.479 & - & - & - \\
\hline Body mass index $\left(\mathrm{kg} / \mathrm{m}^{2}\right)$ & $27.1(4.3)$ & $30.2(5.3)$ & 0.002 & $25.8(3.5)$ & $26.8(3.1)$ & 0.238 \\
\hline $\mathrm{SBP} \mathrm{mmHg}$ & $124.9(\mid 5.5)$ & I33.3 (19.3) & 0.02 & $120.2(9.5)$ & I I I.7 (9.9) & 0.001 \\
\hline $\mathrm{DBP} \mathrm{mmHg}$ & $75.3(9.9)$ & $80.6(10.8)$ & 0.014 & $77.1(5.5)$ & $72.2(7.9)$ & 0.01 \\
\hline Pulse pressure $\mathrm{mmHg}$ & $49.6(11.6)$ & $52.7(13.2)$ & 0.213 & $43.1(7.6)$ & $39.5(7.7)$ & 0.075 \\
\hline $\mathrm{TC}(\mathrm{mmol} / \mathrm{l})$ & $4.6(0.8)$ & $4.9(1.1)$ & 0.234 & $5.0(0.7)$ & $4.8(0.4)$ & 0.227 \\
\hline Triglycerides (mmol/l) & I.3 (0.6) & $\mathrm{I} .2(0.4)$ & 0.462 & $1.6(0.5)$ & I.I (0.4) & $<0.001$ \\
\hline LDL (mmol/l) & $2.8(0.8)$ & $3.1(1.0)$ & 0.137 & $2.7(0.7)$ & $2.9(0.6)$ & 0.133 \\
\hline HDL (mmol/l) & I.2(0.3) & $\mathrm{I} .2(0.4)$ & 0.991 & I.5 (0.4) & I.3 (0.4) & 0.192 \\
\hline $\mathrm{FBG}(\mathrm{mmol} / \mathrm{l})$ & $8.2(3.9)$ & $9.3(4.1)$ & 0.19 & $4.8(0.5)$ & $4.7(0.5)$ & 0.548 \\
\hline eGFR (mls/min) & $105.9(26.8)$ & $78.9(25.4)$ & $<0.001$ & $110.8(12.3)$ & I08.7 (I I.4) & 0.739 \\
\hline eGFR <60 mls/min n(\%) & $19(40.4)$ & 27 (50.9) & 0.321 & - & - & - \\
\hline
\end{tabular}

Data expressed as means (standard deviation)

DM, diabetes mellitus; SBP, systolic blood pressure; DBP, diastolic blood pressure; TC, total cholesterol; LDL, low density lipoprotein cholesterol; HDLC, high density lipoprotein cholesterol; FPG, fasting plasma glucose; eGFR, estimated glomerular filtration rate

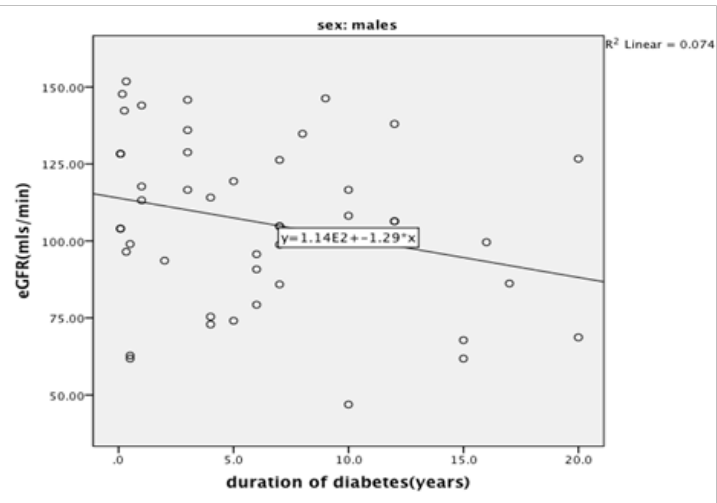

Figure I Correlation between duration of DM and estimated glomerular filtration rate among male patients with diabetes.

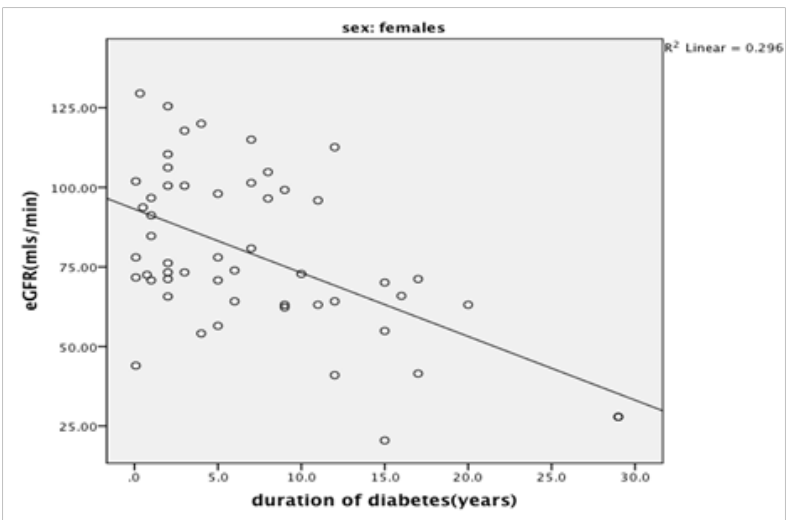

Figure 2 Correlation between duration of DM and estimated glomerular filtration rate among female patients with diabetes.

Table 3 Factors predicting eGFR among the diabetic patients

\begin{tabular}{|c|c|c|c|c|c|}
\hline \multirow{2}{*}{ Model } & \multicolumn{2}{|c|}{ Unstandardized coefficients } & \multirow{2}{*}{$\begin{array}{l}\text { Standardized coefficients } \\
\text { Beta }\end{array}$} & \multirow{2}{*}{$\mathbf{t}$} & \multirow{2}{*}{ Sig. } \\
\hline & B & Std. error & & & \\
\hline (Constant) & 204.873 & 19.727 & & 10.386 & 0 \\
\hline BMI & 0.511 & 0.459 & 0.088 & 1.115 & 0.268 \\
\hline SBP $(\mathrm{mmHg})$ & 0.084 & 0.181 & 0.052 & 0.466 & 0.642 \\
\hline $\mathrm{DBP}(\mathrm{mmHg})$ & -0.116 & 0.299 & -0.043 & -0.389 & 0.698 \\
\hline Female Sex & -31.594 & 4.623 & -0.542 & -6.834 & 0 \\
\hline Age (years) & -1.534 & 0.22 & -0.545 & -6.987 & 0 \\
\hline
\end{tabular}

Coefficients $^{\mathrm{a}}$

a, Dependent Variable: eGFR ( $\mathrm{mls} / \mathrm{min})$ 
With regards to achieving targets for risk factor control (Table 4), overall less than one third of the total number of patients with DM were at the recommended target for BMI. Fewer women compared to men achieved this but the difference was not statistically significant. Thirty five percent of the DM patients were at the recommended target for fasting blood glucose and significantly fewer women compared to men achieved this $(\mathrm{p}=0.005)$. This was despite there being no statistically significant difference in the use of glucose-lowering treatment in both sexes (Table 5). Thirty four percent achieved LDL target with no significant difference between males and females. On the other hand, although a relatively large proportion achieved the targets for BP (62\% of total DM patients), HDL $(60 \%$ of total DM patients) and TG ( $82 \%$ of total DM patients), significantly fewer women achieved the BP and HDL targets compared to men (Table 4).

Table 4 Proportion of patients with DM that was at target for risk factor control (IDF 20I2)

\begin{tabular}{lllll}
\hline Variable n (\%) & $\begin{array}{l}\text { All DM } \\
\text { patients } \\
\mathbf{n = 1 0 0}\end{array}$ & $\begin{array}{l}\text { Males } \\
\mathbf{n = 4 7}\end{array}$ & $\begin{array}{l}\text { Females } \\
\mathbf{n}(\%) \mathbf{n = 5 3}\end{array}$ & P value \\
\hline $\mathrm{BMI}<25 \mathrm{~kg} / \mathrm{m}^{2}$ & $27(27)$ & $16(34.0)$ & $\mathrm{II}(20.8)$ & 0.177 \\
$\mathrm{BP} \leq 130 / 80 \mathrm{mmHg}$ & $62(62)$ & $36(76.7)$ & $26(49.1)$ & 0.007 \\
$\mathrm{FPG}<6.5 \mathrm{mmol} / \mathrm{l}$ & $35(35)$ & $23(48.9)$ & $12(22.6)$ & 0.005 \\
$\mathrm{LDL}<2 \mathrm{mmol} / \mathrm{I}$ & $34(34)$ & $16(34.0)$ & $18(34.0)$ & $\mathrm{I}$ \\
$\mathrm{HDL}>1 \mathrm{mmol} / \mathrm{l}$ & $60(60)$ & $34(72.3)$ & $26(49.1)$ & 0.015 \\
$\mathrm{TG}<2.3 \mathrm{mmol} / \mathrm{l}$ & $82(82)$ & $38(80.9)$ & $44(83.0)$ & 0.49 \\
\hline
\end{tabular}

Data expressed as numbers (percentages).

BMI, body mass index; BP, blood pressure; LDL, low density lipoprotein cholesterol; HDL, high density lipoprotein cholesterol; FPG, fasting plasma glucose; TG, triglycerides

Table 5 Treatment of diabetes mellitus

\begin{tabular}{llll}
\hline Treatment & Males & Females & P value \\
\hline Diet alone & $0(0)$ & $\mathrm{I}(1.9)$ & \\
Oral hypoglycemic drugs $(\mathrm{OHA})$ & $33(70.2)$ & $34(64.2)$ & \\
OHA plus insulin & $\mathrm{II}(23.4)$ & $10(18.9)$ & 0.394 \\
Insulin & $3(6.4)$ & $8(15.1)$ & \\
\hline
\end{tabular}

Data presented as number (percentages)

\section{Discussion}

In this cross sectional study, we showed that compared to individuals who do not have T2DM, patients with T2DM were older, had higher body mass index, systolic and diastolic blood pressures, lower HDL and eGFR. These findings are in keeping with previous large-scale epidemiologic studies that have shown that risk factors for CVD are more prevalent among patients with diabetes compared to those without this condition. ${ }^{12}$

Similar to the findings by Göbl et al. ${ }^{13}$ women in this study when compared to men had higher systolic and diastolic BP and were less likely to achieve BP targets. It is noteworthy that among the control population without DM, the women had significantly lower systolic and diastolic blood pressures compared to age matched men, as is the expected pattern. ${ }^{14}$ High blood pressure especially in the presence of DM significantly increases cardiovascular risk therefore getting women to BP targets is very important and more aggressive measures may be required. A meta-analysis of 37 cohort studies showed that women with DM have a risk of death from CVD that is $50 \%$ higher than that of their male counterparts. ${ }^{15}$ In addition a more recent metaanalysis including over eight hundred thousand individuals showed that the risk of new onset coronary heart disease was $44 \%$ higher in women with DM compared to men. This was independent of confounders like age, BMI, blood pressure and lipids. ${ }^{16}$

In the current study significantly fewer women with DM achieved glycaemic control compared to the men. The difference in glycaemic control was noted despite similar proportions of men and women treated in similar fashion. Biologic explanation for this may include the fact that estrogen, which normally enhances insulin secretion and thus reduces glycaemia, has been shown to decline even before menopause in women with diabetes. ${ }^{17,18}$ In a cross-sectional study involving 3849 individuals with diabetes, Wexler et al. ${ }^{19}$ showed that women were less likely to achieve blood pressure and glycaemic targets (defined as Glycated Haemoglobin levels less than 7\%). This was irrespective of whether they had coronary heart disease or not. This was attributed to differences in clinical treatment the patients received as the women were found to have been less aggressively treated when compared to men.

The women in this study had higher mean TC, and LDL with lower mean TG. Although these did not reach statistical significance, the small sample size may have limited the ability to detect significant differences. In terms of proportions meeting targets, fewer women met the target for high HDL. This is in contrast to the expectation that women should have higher HDL levels than men. ${ }^{20}$ In the study by Wannamethee et al..$^{21}$ they also found that women with diabetes had lower HDL values than their male counterparts in addition to having a less favorable cardiovascular risk profile with higher waist circumference and diastolic blood pressure. They attributed these gender differences to increased insulin resistance in the women associated with adiposity.

Although there was no significant difference in the prevalence of CKD $\left(<60 \mathrm{ml} / \mathrm{min} / 1.73 \mathrm{~m}^{2}\right)$ between men and women in this study, the latter had a lower mean eGFR compared to men and in regression analysis, female gender was a significant predictor of declining GFR independent of blood pressure and BMI. The beneficial effects of the female sex hormones especially estrogen on the kidneys contribute to the lower risk that non-diabetic women have for renal disease compared to non-diabetic men..$^{22}$ However among diabetic women, estrogen levels decline even before menopause and this may provide some pathophysiological explanation for renal disease among women with diabetes. Gender differences in the incidence and prevalence of renal impairment in diabetes vary in various studies. In a UK study of over three thousand patients with diabetes, fewer men compared to women had eGFR $<60 \mathrm{ml} / \mathrm{min} / 1.73 \mathrm{~m}^{2}{ }^{23}$ Similarly data from the UKPDS showed that females had a higher risk of CKD compared to men. ${ }^{24}$ On the other hand, Yu et al., in their analysis of data from the Pathways study, found that men with diabetes had a higher prevalence of diabetic kidney disease however; women were more likely to have advanced diabetic kidney disease. ${ }^{25}$

Hypertension, hyperglycemia and dyslipidemia are all important risk factors for CVD and the finding in this study that fewer women were at target for these parameters raises the question as to the mechanisms accounting for these differences. Women, while more likely to utilize health care facilities and be compliant with medication, may be less aggressively treated than their male counterparts due 
to the preconceived notion by healthcare practitioners and patients themselves that cardiovascular disease is a 'man's disease'. It may well also reflect the fact that treatments available may not work in the same way in women as they do in men or the pathophysiologic processes underling these conditions are different in women. In this study the women with DM had a significantly higher mean BMI than men. Obesity, which is a major risk factor for diabetes, is associated with insulin resistance, hyperglycemia and higher blood pressure so this may be a key risk factor that underscores the gender differences noted in this study. As Huxley et al. ${ }^{15}$ noted in their meta-analysis women may need to put on more weight compared to men to get diabetes and thus are more likely to spend a longer time with adverse metabolic profile prior to a formal diagnosis of diabetes.

\section{Limitations}

The small sample size limits the ability to detect significant differences. The cross-sectional design does not allow for precise determination of the influence of gender on decline in kidney function. The blood pressure- and cholesterol lowering medications used were not included in the analysis. Glycated hemoglobin which is a better way to assess glucose control was not used in this study therefore glycemic control may not reflect true glycaemia in this population. However this study highlights some important differences in cardiovascular risk factors and a significant association between female gender and declining eGFR. Further studies are warranted to elucidate the pathophysiologic mechanisms linking this association.

\section{Conclusion}

This study has highlighted that overall risk factor control in a hospital population of black Nigerians is sub-optimal with females being worse off compared to their male counterparts. There was also an association with female gender and decline in eGFR. This emphasizes the necessity for implementation of guideline recommendations for better risk factor control among patients with diabetes mellitus, especially among women.

\section{Authors contribution}

Both authors designed the study. CW carried out data collection, $\mathrm{SO}$ analyzed the data and both authors prepared the final draft.

\section{Acknowledgements}

None.

\section{Conflict of interest}

Author declares that there is no conflict of interest.

\section{References}

1. International Diabetes Federation (IDF). IDF Diabetic Atlas. 5th ed Belgium; 2014.

2. Ugoya SO, Ugoya TA, Puepet FH, et al. Risk determination of diabetic peripheral neuropathy in Jos North Central Nigeria. J Chinese Clinical Medicine. 2008;3(5):1-5.

3. Akinkugbe OO. Non-communicable diseases in Nigeria- Final report of a National Survey. Federal Ministry of Health-National Expert Committee on Non-Communicable Diseases. Lagos, Nigeria; 1997. p. 1-12.

4. Nyenwe EA, Odia OJ, Ihekwaba AE, et al. Type 2 diabetes in adult Nigerians: a study of its prevalence and risk factors in Port Harcourt Nigeria. Diabetes Res Clin Pract. 2003;62(3):177-185.
5. Pilote L, Dasgupta K, Guru V, et al. A comprehensive view of sex-specific issues related to cardiovascular disease. CMAJ. 2007;176(6):S1-S44.

6. Stratton IM, Adler AI, Neil HAW, et al. Association of glycaemia with macrovascular and microvascular complications of type 2 diabetes (UKPDS 35): prospective observational study. $B M J$ 2000;321(7258):405-412.

7. Rydén L, Standl E, Bartnik M, et al. Guidelines on diabetes, prediabetes, and cardiovascular diseases: executive summary The Task Force on Diabetes and Cardiovascular Diseases of the European Society of Cardiology (ESC) and of the European Association for the Study of Diabetes (EASD). Eur Heart J. 2007;28(1):88-136.

8. Chobanian AV, Bakris GL, Black HR. The Seventh Report of the Joint National Committee on Prevention, Detection, Evaluation, and Treatment of High Blood Pressure: The JNC 7 report. JAMA. 2003;289(19):2560-2572.

9. Cockcroft DW, Gault MH. Prediction of creatinine clearance from serum creatinine. Nephron. 1976;16(1):31-41.

10. Friedwald WT, Levy RI, Fredrickson DS. Estimation of the Concentration of Low Density Lipoprotein Cholesterol in Plasma without Use of Preparative Ultra centrifugation. Clin Chem. 1972;18(6):499-502.

11. International Diabetes Federation. clinical guidelines taskforce. Global Guideline for Type 2 Diabetes. 2012.

12. Hurst RT, Lee RW. Increased incidence of coronary atherosclerosis in type 2 diabetes mellitus: mechanisms and management. Ann Intern Med. 2003;139(10):824-834.

13. Gobl CS, Brannath W, Bozkurt L, et al. Sex-specific differences in glycemic control and cardiovascular risk factors in older patients with insulin-treated type 2 diabetes mellitus. Gend Med. 2010;7(6):593-599.

14. Lewington S, Clarke R, Qizilbash N, et al. Age-specific relevance of usual blood pressure to vascular mortality: a meta-analysis of individual data for one million adults in 61 prospective studies. Lancet. 2002;360(9349):1903-1913.

15. Huxley R, Barzi F, Woodward M. Excess risk of fatal coronary heart disease associated with diabetes in men and women: meta- analysis of 37 prospective cohort studies. BMJ. 2006;332(7533):73-78.

16. Peters SAE, Huxley RR, Woodward M. Diabetes as risk factor for incident coronary heart disease in women compared to men: a systematic review and meta-analysis of 64 cohorts including 858,507 individuals and 28,203 coronary events. Diabetologia. 2014;57(8):1542-1551.

17. Stamataki KE, Spina J, Rangou DB, et al. Ovarian function in women with non-insulin dependent diabetes mellitus. Clin Endocrinol (Oxf). 1996;45(5):615-621.

18. Ding EL, Song Y, Malik VS, et al. Sex differences of endogenous sex hormones and risk of type 2 diabetes: a systematic review and metaanalysis. JAMA. 2006;295(11):1288-1299.

19. Wexler DJ, Grant RW, Meigs JB, et al. Sex disparities in treatment of cardiac risk factors in patients with type 2 diabetes. Diabetes Care. 2005;28(3):514-520

20. O'Meara JG, Kardia SL, Armon JJ, et al. Ethnic and sex differences in the prevalence, treatment, and control of dyslipidemia among hypertensive adults in the GENOA study. Arch Intern Med. 2004;164(12):1313-1318.

21. Wannamethee SG, Papacosta O, Lawlor DA, et al. Do women exhibit greater differences in established and novel risk factors be- tween diabetes and non-diabetes than men? The British Regional Heart Study and British Women's Heart Health Study. Diabetologia. 2012;55(1):80-87.

22. Ix JH, Chonchol M, Laughlin GA, et al. Relation of sex and estrogen therapy to serum fibroblast growth factor 23, serum phosphorus, and urine phosphorus: the Heart and Soul Study. Am J Kidney Dis. 2011;58(5):737-745. 
23. Nag S, Bilous R, Kelly W, et al. All-cause and cardiovascular mortality in diabetic subjects increases significantly with reduced estimated glomerular filtration rate (eGFR): 10years' data from the South Tees Diabetes Mortality study. Diabet Med. 2007;24(1):10-17.

24. Retnakaran R, Cull CA, Thorne KI, et al. Risk factors for renal dysfunction in type 2 diabetes: U.K. Prospective Diabetes Study 74. Diabetes. 2006;55(6):1832-1839.
25. Yu KM, Lyles CR, Bent-Shaw LA, et al. Risk factor, age and sex differences in chronic kidney disease prevalence in a diabetic cohort: The Pathways Study. Am J Nephrol. 2012;36(3):245-251. 